\title{
Revista \\ Brasileira \\ de Enfermagem \\ REBEn \\ Uso de medicamentos, glicemia capilar e índice de massa corpórea em pacientes com diabetes mellitus
}

PESQUISA

\author{
Drug use, blood glucose and body mass index in patients with diabetes mellitus \\ Uso de fármacos, glucemia e índice de masa corporal en pacientes con diabetes mellitus
}

\begin{abstract}
Márcio Flávio Moura de Araújo', Thiago Moura de Araújo', Priscila de Jesus dos Santos Alves', Vivian Saraiva Veras", Maria Lúcia Zanetti", Marta Maria Coelho Damasceno'

' Universidade Federal do Ceará, Faculdade de Farmácia Odontologia e Enfermagem, Departamento de Enfermagem. Programa de Pós-Graduação em Enfermagem. Fortaleza-CE, Brasil.

"Universidade de São Paulo, Escola de Enfermagem de Ribeirão Preto, Programa de Pós-Graduação em Enfermagem. Ribeirão Preto-SP, Brasil.
\end{abstract}

Submissão: 16-06-2011 Aprovação: 01-07-2013

\section{RESUMO}

Objetivou-se relacionar o uso de medicamentos com a glicemia capilar e o índice de massa corpórea em pacientes com diabetes mellitus tipo 2. Estudo transversal realizado entre janeiro e julho de 2009, nos domicílios de 437 pacientes de doze centros de saúde de Fortaleza-CE. Utilizou-se um formulário para o registro das variáveis sociodemográficas e clinicas, glicemia capilar, índice de massa corpórea e uso de medicamentos. Dos pacientes com glicemia capilar normal, 93\% não utilizavam antidepressivos / ansiolíticos $(p=0,02)$. Aproximadamente $99 \%$ dos que apresentavam a glicemia capilar elevada não utilizavam cálcio diariamente $(p=0,04)$. Entre aqueles com índice de massa corpórea normal, 45,5\% tomavam diariamente anti-hipertensivos da classe inibidor da ECA $(p=0,03)$. O uso diário de drogas inibidoras da ECA, antidepressivos / ansiolíticos e cálcio apresentou associação com a glicemia capilar e o índice de massa corpórea, respectivamente. É importante que o enfermeiro avalie o peso corporal e a glicemia capilar dos pacientes com diabetes, em particular daqueles que fazem uso contínuo de medicamentos.

Descritores: Diabetes Mellitus Tipo 2; Drogas; Glicemia; Índice de Massa Corporal; Enfermagem.

\begin{abstract}
This research aimed to relate medication use with blood glucose and body mass index in subjects with type 2 diabetes mellitus. It was performed a cross-sectional study, between January and July 2009, at the homes of 437 users of 12 health centers in FortalezaCE. We administered a survey about blood glucose, body mass index and medication use. Approximately $99 \%$ of study subjects with altered glucose classified as not using daily calcium $(\mathrm{p}=0.04)$. Among those with normal blood glucose levels classified as around 93\% did not use daily antidepressants / anxiolytics ( $p=0.02$ ). Among those with DM 2 normal body mass index, $45.5 \%$ were using daily anti-hypertensive ACE-inhibitor class ( $p=0.03$ ). Daily use of ACE inhibitor drugs, antidepressants / anxiolytics and calcium was associated with body mass index and glucose, respectively. It is important for nurses to assess body weight and blood glucose in patients with diabetes, particularly those that are under continuous medication.
\end{abstract}

Key words: Diabetes Mellitus Type 2; Drugs; Blood Glucose; Body Mass Index; Nursing.

\section{RESUMEN}

Objetivó-se relacionar el uso de medicamentos con la glucemia y índice de masa corporal en pacientes con diabetes mellitus tipo 2. Fue realizado un estudio transversal, entre enero y julio de 2009, en las casas de 437 usuarios de 12 centros de salud en Fortaleza-CE. Se administró una encuesta sobre la glucemia, índice masa corporal y el uso de medicamentos. Aproximadamente $99 \%$ de los sujetos con glucemia clasificada como alterada no usaban diariamente calcio $(p=0,04)$. Entre las personas con niveles de glucemia clasificados como normales, $93 \%$ no utilizaban antidepresivos / ansiolíticos $(p=0,02)$. Entre las personas con índice de masa corporal normal, 45,5\% usaban inhibidor de la ECA contra la hipertensión ( $p=0,03$ ). El uso diario de fármacos inhibidores de la ECA, antidepresivos / ansiolíticos y el calcio se asoció con el índice de masa corporal y la glucemia, respectivamente. Es importante que los enfermeros controlen continuamente la situación nutricional y los niveles de glucosa de las personas con diabetes, especialmente los que utilizan múltiplos fármacos simultáneamente.

Palabras clave: Diabetes Mellitus Tipo 2; Drogas; Glucemia; Índice de Masa Corporal; Enfermería. 


\section{INTRODUÇÃO}

A maior parte dos pacientes com Diabetes Mellitus tipo 2 (DM2) está com excesso de peso, pressão arterial elevada e/ ou dislipidemias. Em virtude disto e do caráter crônico da doença, de modo geral, as pessoas serão incapazes de conseguir prevenir complicações sem a adoção de medicamentos como antidiabéticos orais (AO), anti-hipertensivos e antilipêmicos ${ }^{(1)}$. Neste caso, os pacientes podem estar inseridos em situações de polifarmácia, quando cinco ou mais medicamentos são utilizados continuamente e passam a ser um problema de saúde pública. A polifarmácia está associada ao aumento do risco e da gravidade das reações medicamentosas adversas, de precipitar interações farmacológicas, ou ainda causar toxicidade cumulativa, ocasionar erros na tomada das drogas e reduzir a adesão ao tratamento ${ }^{(2)}$.

Pacientes que utilizam antidiabéticos orais (ADO) estão susceptíveis a interações medicamentosas com outras drogas, sendo algumas dessas interações já conhecidas na literatura. Dentre as interações medicamentosas que aumentam a toxicidade dos ADO sobressaem às associações com cloranfenicol (50\%), cimetidina (34\%), propranolol (34\%), anti-inflamatório não esteroidal (34\%) e inibidores da monoaminoxidase (34\%). Das que diminuem a eficácia dos ADO, as associações mais frequentes ocorrem com corticosteroides (83\%), diuréticos $(83 \%)$, contraceptivos orais $(83 \%)$ e fenotiazidas $(67 \%)$. Observa-se a possibilidade de interferência na eficácia terapêutica e consequentemente no manejo adequado do controle glicêmico ao se associar determinadas drogas ao uso de $\mathrm{ADO}^{(3)}$.

Tal constatação é preocupante já que algumas doenças estão relacionadas ao DM2. Por exemplo, aproximadamente $70 \%$ dos pacientes com DM2 são hipertensos e, nesse caso, a terapêutica farmacológica anti-hipertensiva, conforme as drogas adotadas, pode ser um agravante do regime glicêmico satisfatório $^{(4-5)}$. O tratamento da hipertensão arterial em sujeitos com DM 2 reduz a mortalidade cardiovascular e retarda o declínio da função glomerular. Entretanto, a escolha dos agentes anti-hipertensivos no tratamento de pacientes nestas condições deve levar em consideração os efeitos das drogas sobre o peso corporal, a sensibilidade insulínica, o perfil lipídico e complicações do DM 2 ou da hipertensão arterial $^{(6)}$.

Pesquisas desenvolvidas nos Estados Unidos da América e na Europa argumentam que a maior parte dos fármacos anti-hipertensivos parece exercer diferentes efeitos no controle glicêmico. Diuréticos tiazídicos e betabloqueadores são potencialmente diabetogênicos; já os bloqueadores dos canais de cálcio parecem ser neutros e os inibidores do sistema renina-angiotensina estão relacionados com um melhor controle e até prevenção do $\mathrm{DM} 2^{(6-8)}$.

Outra doença inerente ao diabetes é a depressão; cerca de 20 a $30 \%$ dos pacientes desenvolvem este problema de saúde mental. O tratamento farmacológico da depressão nos portadores desta endocrinopatia é outra terapêutica possível de prejudicar o controle glicêmico. De modo geral, os antidepressivos tricíclicos, em especial os com maior ação noradrenérgica, e os inibidores da monoaminoxidase tendem a aumentar os níveis glicêmicos e piorar o controle metabólico dos pacientes ${ }^{(9)}$. Uma pesquisa realizada com 3.234 participantes com sobrepeso e intolerância a glicose concluiu que há riscos para o controle metabólico da doença no uso desses psicofármacos. Embora o estudo não tenha sido suficiente para mostrar quais antidepressivos podem causar estas alterações no diabetes, os resultados apontam que isto não se deve à elevação de peso, efeito colateral de muitos antidepressivos, ou como resultados de alteração na glicemia de jejum ou dos níveis de insulina ${ }^{(10)}$.

Diante deste panorama, um dos grandes desafios dos profissionais de saúde, entre eles o enfermeiro, que cuidam de pacientes com DM2, sobretudo os idosos, é conhecer a interação entre ADO e demais drogas ou ainda a interferência de determinadas drogas no controle glicêmico dos pacientes. Uma vez estabelecida essas interações, o enfermeiro poderá contribuir para a promoção do uso racional de medicamentos sem agravar o controle metabólico da doença, mediante orientações rigorosas para monitorização da glicemia capilar, peso corporal e primeiros sinais de complicações micro e macrovasculares. Portanto, o objetivo deste estudo foi relacionar o uso de medicamentos com a glicemia capilar e o índice de massa corpórea em pacientes com DM2.

\section{MÉTODO}

Trata-se de um estudo transversal desenvolvido de janeiro a setembro de 2009 em doze Centros de Saúde da Família situados em Fortaleza-CE, Brasil. Essa metrópole está dividida em seis regiões geoeconômicas e possuía 173mil usuários com diabetes cadastrados em sua rede básica de saúde em 2008. Dessa forma, para obter dados representativos em torno do assunto nesta cidade, foram escolhidos, por conveniência, doze serviços de atenção em DM2, sendo dois em cada uma das seis regiões municipais. As unidades de saúde selecionadas localizavam-se em bairros distintos no respeitante a infraestrutura, serviços e situação socioeconômica.

A amostra foi distribuída por conglomerados e, para o seu cálculo, utilizou-se fórmula para populações infinitas. A variável uso de ADO entre pacientes com DM2 na atenção básica foi escolhida como desfecho e sua prevalência adotada foi de $60 \%{ }^{(11)}$, enquanto o nível de significância e o erro amostral foram $(=0,05)$ e $5 \%$, respectivamente. O tamanho da amostra ficou estabelecido em 369 com diabetes. Sobre este número foi acrescida uma taxa de $10 \%$, considerando perdas de dados nas quais foram incluídas recusas; hospitalizações; desistências; informações erradas e/ou não disponíveis. Assim, a amostra foi constituída por 437 pacientes com DM2.

Os pacientes foram selecionados de maneira não probabilística dentro de cada serviço de saúde escolhido desde que atendessem aos seguintes critérios de inclusão: ter diagnóstico confirmado de DM 2 registrado no prontuário ou ficha de saúde; ser atendido (a) nos serviços da rede de atenção primária selecionados para o estudo; residir em Fortaleza-Brasil e possuir telefone para contato; estar em tratamento com ADO; estar, há pelo menos seis meses com a mesma prescrição a fim de evitar mudanças na terapêutica medicamentosa passíveis de interferir no estudo e ter prontuário ou ficha de saúde disponível no serviço. Como critérios de exclusão, constaram: 
estar em tratamento combinado com ADO e insulina; ficar hospitalizado no período da coleta de dados e ser dependente de outras pessoas para tomar os ADO.

Promoveram-se visitas domiciliárias para a coleta de informações referentes ao tratamento medicamentoso, as quais foram realizadas durante o período de fevereiro a julho de 2009 nos turnos matutino e vespertino em conjunto com agentes comunitários de saúde. Para a coleta de dados construiu-se um formulário com informações referentes às variáveis sociodemográficas e clínicas, glicemia capilar, índice de massa corpórea (IMC) e uso de medicamentos.

Mensurou-se a glicemia capilar mediante a utilização de glicosímetro e da respectiva fita-teste da marca Optium ${ }^{\circledast}$. Os valores detectados levaram em consideração o estado de jejum ou pós-prandial dos pacientes. Dessa maneira, os resultados foram interpretados da seguinte forma naqueles em jejum: 70-110 mg/ dl (Bom), 111-140 mg/dl (Aceitável) e > 140 mg/dl (Insatisfatório). Já nos pacientes alimentados, a classificação foi a seguinte: 70-140 mg/dl (Bom), 141-160 mg/dl (Aceitável) e > 160 mg/dl (Insatisfatório). Entretanto, para fins estatísticos, as classificadas como bom e aquelas com valor aceitável ou insatisfatório foram denominadas normal e elevada, respectivamente ${ }^{(12)}$.

Para a verificação do IMC utilizou-se o cálculo de Índice de Massa Corporal (IMC), por meio da fórmula [peso $(\mathrm{kg}) /$ altura $\left.\left(\mathrm{m}^{2}\right)\right]$. Os dados antropométricos dos entrevistados foram autorreferidos e em seguida confrontados com os respectivos dados dos cartões de saúde no domicílio ${ }^{(13)}$.

No processamento destas informações trabalhou-se com o software Epi-Info versão 6.04. O intervalo de confiança adotado foi de $95 \%$. Na análise da relação entre o uso de medicamentos com a glicemia capilar e o índice de massa corpórea empregou-se o teste exato de Fischer, quando apropriado, e do Qui-quadrado. O projeto foi aprovado pelo Comitê de Ética em Pesquisa do Centro de Ciências da Saúde da Universidade Federal do Ceará, protocolo 47/09. Todos os participantes assinaram o Termo de Consentimento Livre e Esclarecido.

\section{RESULTADOS}

Dos 437 pacientes, (70,3\%) eram mulheres e (48,1\%), brancos. Quanto à faixa etária, encontraram-se sujeitos entre $18 \mathrm{e}$ 92 anos de idade, distribuídos nas seguintes faixas etárias: 1859 anos (41,1\%), 60-69 anos (29,5\%), 70-79 anos (20,1\%) e 80-92 anos (9,3\%). A média de idade e de anos de estudo foi de 63,1 $\left(\mathrm{DP}_{ \pm} 11,6\right)$ e 4,3 (DP $\left.\pm 3,7\right)$ anos, respectivamente. Em relação ao estado civil, (55,8\%) eram casados ou viviam numa relação estável, enquanto $6,9 \%$ eram solteiros. Parcela substancial tinha casa própria $(84,9)$ e, $(45,8 \%)$ viviam em família nuclear composta por companheiro, filhos e/ou netos. Ademais, $(49,4 \%)$ eram aposentados. A aposentadoria era a fonte de renda principal de sustentação da prole $(48,7 \%)$. Houve um predomínio das classes econômicas $\mathrm{D}$ e C e a média da renda familiar mensal era de $\bigcup \$ 445,9$ dólares $\left(\mathrm{DP}_{ \pm} \mathbf{3 2 7 , 1}\right.$ ).

O IMC obtido foi distribuído da seguinte maneira, a saber: sobrepeso $(35,7 \%)$, eutrófico $(24,7 \%)$, obesidade $1(20,8 \%)$, obesidade $2(6,2 \%)$, obesidade $3(2,3 \%)$ e baixo peso $(2,3 \%)$.

Os ADO mais usados eram metformina $(22,9 \%)$, glibenclamida (21,5\%) e a associação destas (43,5\%), com uma média de tomada de 3,0 (DP $\pm 1,7)$ comprimidos diários. Parte expressiva dos pacientes tinha comorbidades associadas ao DM $2(79,8 \%)$ e usava medicamentos para tratá-las $(71,7 \%)$, em média tomavam $4,2(\mathrm{DP} \pm 3,6)$ comprimidos ao dia. As principais comorbidades identificadas foram hipertensão arterial $(72,9 \%)$, doença cardiovascular (11,2\%) e problemas ósseos e/ou articulares (6,9\%).

Tabela 1 - Distribuição dos pacientes com DM2 segundo o tipo de medicamento utilizado e os valores da glicemia capilar. Fortaleza-CE, Brasil. 2009.

\begin{tabular}{|c|c|c|c|c|c|}
\hline \multirow[t]{2}{*}{ Drogas } & \multicolumn{4}{|c|}{ Glicemia } & \multirow[t]{2}{*}{ Valor $p$} \\
\hline & & Normal & & vada & \\
\hline & $\mathbf{n}$ & $\%$ & $\mathbf{n}$ & $\%$ & \\
\hline \multicolumn{6}{|l|}{ Antidepressivos/ansiolíticos } \\
\hline Sim & 08 & 7,2 & 08 & 2,5 & $0,023 *$ \\
\hline Não & 104 & 92,8 & 317 & 97,5 & \\
\hline \multicolumn{6}{|l|}{ Cálcio } \\
\hline Sim & 06 & 5,4 & 06 & 1,8 & \\
\hline Não & 106 & 94,6 & 319 & 98,2 & $0,04 *$ \\
\hline \multicolumn{6}{|l|}{ Corticoides } \\
\hline Sim & 03 & 2,7 & 05 & 1,5 & \\
\hline Não & 109 & 97,3 & 320 & 98,5 & $0,33^{*}$ \\
\hline \multicolumn{6}{|l|}{ Inibidor da ECA } \\
\hline Sim & 69 & 61,6 & 170 & 52,3 & \\
\hline Não & 43 & 38,4 & 155 & 47,7 & $0,11 * *$ \\
\hline \multicolumn{6}{|l|}{ Diurético tiazídico } \\
\hline Sim & 47 & 41,1 & 102 & 31,5 & \\
\hline Não & 66 & 58,0 & 222 & 68,5 & $0,08 * *$ \\
\hline \multicolumn{6}{|l|}{ Betabloqueador } \\
\hline Sim & 16 & 14,3 & 46 & 14,2 & \\
\hline Não & 96 & 85,7 & 279 & 85,8 & $0,90 * *$ \\
\hline
\end{tabular}

\footnotetext{
* Teste Exato de Fischer ** Teste do Qui-quadrado
} 
Os anti-hipertensivos utilizados eram inibidor da ECA-enzima conversora de angiotensina (52,8\%), diurético tiazídico $(32,4 \%)$ e inibidor adrenérgico central $(18,5 \%)$. Cabe destacar ainda o uso de antiagregante plaquetário (29,9\%).

A Tabela 1 mostra a distribuição dos pacientes com DM2 conforme os medicamentos utilizados e a glicemia capilar. Nota-se que houve associação estatisticamente significante entre uso de antidepressivos/ansiolíticos e cálcio e glicemia capilar elevada.

Parcela substancial da amostra estava com a glicemia capilar elevada $(86,2 \%)$ e com excesso de peso $(79,6 \%)$. De acordo com os resultados aproximadamente $99 \%$ dos pacientes com glicemia capilar alterada não tomavam cálcio $(p=0,04)$ diariamente. Por sua vez, 93\% dos pacientes com glicemia normal não faziam uso diário de antidepressivos/ansiolíticos $(p=0,02)$. Todavia, na amostra investigada, não foi possível identificar diferenças estatisticamente significantes entre a glicemia e o uso diário de corticoides $(p=0,33)$, inibidor da ECA $(p=0,11)$, diurético tiazídico $(p=0,08)$ e betabloqueador $(p=0,90)$ ( Tabela 1$)$.

A Tabela 2 mostra os pacientes com DM 2 de acordo com o IMC. Daqueles com IMC normal, 45,5\%, utilizavam anti-hipertensivos da classe inibidor da ECA, diariamente $(p=0,03)$. Não foi evidenciada associação estatisticamente significante entre o IMC e o uso de medicamentos como antidepressivos/ansiolíticos, cálcio, corticoide, diurético tiazídico e betabloqueador.

\section{DISCUSSÃO}

A amostra foi constituída, sobretudo, por idosos acima do peso, com comorbidades e em uso de medicamentos para o DM 2, hipertensão arterial, problemas circulatórios e ósseos, entre outros.

Nos Estados Unidos da América, aproximadamente 20\% dos adultos com 65 anos ou mais são acometidos por DM 2. Portanto, são cerca de sete milhões de pessoas. Nesse sentido, cabe pontuar que essa faixa etária futuramente sofrerá um incremento de 12,6\% para 13,2\% nos casos de DM 2. Além disso, essas pessoas terão outras complicações, especialmente a obesidade e a hipertensão arterial ${ }^{(1)}$. É fato que idosos portadores de doenças crônicas podem chegar a tomar mais de dez comprimidos diários. Diante disto, o risco para o desenvolvimento de interações medicamentosas é estimado em $13 \%, 58 \%$ e $82 \%$ para idosos que usam dois, cinco, sete ou mais medicamentos, respectivamente ${ }^{(14)}$.

A predominância de casos de excesso de peso e de hipertensão arterial detectadas neste estudo foi similar aos achados de outras pesquisas que analisaram a população com DM $2^{(11,15-16)}$. Também não se encontrou associação entre a glicemia capilar e o uso de anti-hipertensivos, mas, outras pesquisas encontraram resultados diferentes.

Tabela 2 - Distribuição dos pacientes com DM2 segundo o tipo de medicamento utilizado e o IMC. Fortaleza-CE, Brasil. 2009.

\begin{tabular}{|c|c|c|c|c|c|}
\hline \multirow{2}{*}{ Drogas } & \multicolumn{4}{|c|}{ IMC } & \multirow[t]{2}{*}{ Valor $p$} \\
\hline & \multicolumn{2}{|c|}{ Normal } & \multicolumn{2}{|c|}{ Excesso de peso } & \\
\hline Antidepressivos/ansiolíticos & $\mathbf{n}$ & $\%$ & $\mathbf{n}$ & $\%$ & \\
\hline Sim & 05 & 3,8 & 11 & 3,7 & \\
\hline Não & 128 & 96,2 & 293 & 96,3 & $0,511^{*}$ \\
\hline \multicolumn{6}{|l|}{ Cálcio } \\
\hline Sim & 04 & 3,5 & 12 & 2,6 & \\
\hline Não & 112 & 96,5 & 299 & 97,4 & $0,369 *$ \\
\hline \multicolumn{6}{|l|}{ Corticoides } \\
\hline Sim & 08 & 6,4 & 04 & 1,3 & \\
\hline Não & 117 & 93,6 & 308 & 98,7 & $0,326^{*}$ \\
\hline \multicolumn{6}{|l|}{ Inibidor da ECA } \\
\hline Sim & 86 & 58,1 & 160 & 55,3 & \\
\hline Não & 62 & 41,9 & 129 & 44,7 & $0,037^{* *}$ \\
\hline \multicolumn{6}{|l|}{ Diurético tiazídico } \\
\hline Sim & 13 & 9,9 & 22 & 92,7 & \\
\hline Não & 119 & 90,1 & 283 & 7,3 & $0,418^{* *}$ \\
\hline \multicolumn{6}{|l|}{ Betabloqueador } \\
\hline Sim & 09 & 7,5 & 51 & 20,5 & \\
\hline Não & 111 & 92,5 & 238 & 79,5 & $0,061^{*}$ \\
\hline
\end{tabular}

* Teste Exato de Fischer ** Teste do Qui-quadrado 
Uma investigação desenvolvida em Araraquara-SP com idosos hipertensos e/ou diabéticos constatou que os que usavam anti-hipertensivos (metildopa e captopril) e anticoagulantes (ácido acetil salicílico) tinham predisposição para hipoglicemia, relacionada à potencialização farmacológica dos $\mathrm{ADO}^{(16)}$.

O consumo de drogas inibidoras da ECA reduz problemas cardiovasculares em 25\% isoladamente, e em até $75 \%$ quando associado a outros anti-hipertensivos e antiplaquetários. Esses medicamentos ainda podem prevenir em 27\% a incidência de novos casos de DM 2, todavia, ainda não há uma explicação desse mecanismo protetor ${ }^{(1)}$.

Ainda não foram encontradas evidencias sobre a associação entre o uso diário de inibidor da ECA ea redução de peso corpóreo em pessoas com DM 2. Contudo, observou-se um aumento na produção de insulina em pessoas com DM 2 em uso de inibidores da ECA, aspecto favorável para o alcance da euglicemia ${ }^{(17)}$.

Em relação ao peso corpóreo, estudo mostrou que inibidores da ECA teriam a capacidade de auxiliar na maturação de adipócitos, impedindo o desvio de lipídios para o fígado, músculos esqueléticos e pâncreas. Outro achado importante é o fato do tecido adiposo ser capaz de sintetizar componentes do sistema renina-angiotensina-aldosterona, implicados no aumento de peso ${ }^{(18)}$. Dessa maneira, é plausível afirmar que o bloqueio da produção desses componentes, por meio de drogas inibidoras da ECA, possa contribuir na prevenção de problemas cardiovasculares, sobretudo a hipertensão arterial e a obesidade, ou ainda ser a terapêutica inicial para hipertensos com excesso de peso e/ou glicemia de jejum alterada.

Praticamente $100 \%$ dos pacientes com glicemia alterada não usavam cálcio diariamente. É possível que outros componentes terapêuticos e o estilo de vida dos pacientes tenham relação mais direta com este achado do que a ausência do cálcio na sua alimentação. Entretanto, o emprego contínuo deste componente já demonstrou melhorar o metabolismo lipídico, a resistência insulínica e o peso corpóreo. O cálcio tem um papel importante na secreção de insulina e consequentemente na euglicemia. Porém, seu consumo diário já tem sido alvo de vários debates sobre o benefício para a sensibilidade insulínica. O consumo diário de cálcio tem relação inversa com doença cardiovascular, obesidade, pressão arterial e resistência a insulina. Somado a isto, sugere-se que concentrações alteradas de cálcio e vitamina D estão associadas com o desenvolvimento do DM $2^{(19)}$.

Pesquisa realizada nos Estados Unidos durante três anos com 314 adultos não diabéticos, em uso diário de vitamina D e cálcio, constatou uma redução da glicemia de jejum estatisticamente significante em comparação ao grupo controle (20).O acompanhamento durante nove anos de uma coorte de 4.372 franceses que consumiam cálcio regularmente também constatou uma redução da glicemia e dos níveis de insulina estatisticamente significante entre as mulheres ${ }^{(19)}$.

Diante dos achados das investigações consultadas é perceptível que há divergência nos resultados, mesmo assim, é importante que o profissional de saúde considere a possibilidade destes medicamentos auxiliarem ou não no controle glicêmico e reforce suas orientações de adesão à terapia farmacológica.

Pelo exposto, percebe-se a relevância de orientar a dieta dos pacientes com DM 2, de modo a incluir alimentos que favoreçam o equilíbrio glicêmico. Todavia, isto encontra obstáculo na escassez de recursos financeiros para a manutenção de uma alimentação saudável, com baixo teor calórico e lipídico. Outro fator agravante é que muitas vezes os insumos disponibilizados nos serviços de saúde gratuitamente são insuficientes para o alcance das metas terapêuticas ${ }^{(11)}$. Dessa forma, a aquisição de outros recursos que poderiam auxiliar no controle glicêmico é negligenciada.

Ainda como observado, mais de $90 \%$ da amostra com a glicemia normal não fazia uso diário de ansiolíticos/antidepressivos. Alguns antidepressivos possuem efeitos sobre a glicemia e requerem atenção especial quando utilizados em pacientes com diabetes. Antidepressivos tricíclicos e tetracíclicos, particularmente aqueles com ação predominantemente noradrenérgica como a imipramina, nortriptilina e maprotilina, podem inibir a liberação de insulina pelo pâncreas e causar aumento da glicemia. Por sua vez, os inibidores seletivos de recaptura de serotonina têm sido associados a uma redução dos níveis de glicemia ${ }^{(9)}$.

\section{CONCLUSÕES}

O DM2 é uma doença preocupante em virtude, sobretudo, dos riscos de desenvolvimento de complicações micro e macrovasculares que diminuem a expectativa e qualidade de vida dos pacientes. No intuito de combater e/ou prevenir isto, hoje, o número e a qualidade de medicamentos prescritos são maiores. Tal fato aumenta a chance de fenômenos como a polifarmácia, interações medicamentosas e reações adversas entre os pacientes.

Como se depreende, a atuação do enfermeiro neste quadro é fundamental, pois além de educar sobre o uso ou auxiliar na administração desses fármacos pode promover o empoderamento dos pacientes. Ademais, ao se levar em conta as implicações da polifarmácia sobre o peso e a glicemia capilar de pessoas com diabetes, o enfermeiro deve aumentar sua vigilância no tocante ao controle metabólico do DM2. Esta iniciativa favorecerá a realização de intervenções de enfermagem específicas e culminará em melhoria da qualidade de vida dos pacientes.

O estudo ora exposto apresenta algumas limitações, a saber: o uso da glicemia capilar, parâmetro com acurácia inferior à glicemia venosa e de dados antropométricos autorreferidos, que apesar de válidos, são menos precisos em relação aos obtidos mediante exame físico. Contudo, trouxe novos achados a um tema pouco explorado em periódicos de enfermagem brasileiros e, assim, outras investigações se fazem necessárias especialmente as de caráter longitudinal que poderão elucidar o papel de determinados compostos farmacológicos sobre o controle metabólico do DM2. 


\section{REFERÊNCIAS}

1. Balkrishan R, Rajagopalan R, Camacho FT, Huston SA, Murray FT, Anderson RT. Predictors of medication adherence and associated health care costs in an older population with type 2 diabetes mellitus: a longitudinal cohort study. Clin Ther 2003;25(11):2958-71.

2. Secoli SR. Polifarmácia: interações e reações no uso de medicamentos por idosos. Rev Bras Enferm 2010;63(1):136-40.

3. Santos DB. Interações medicamentosas em pacientes com diabetes mellitus tipo 2. São Paulo. Monografia [Especialização em Enfermagem] - Escola de enfermagem da USP; 2008.

4. Ministério da Saúde. Secretaria de Atenção à Saúde. Caderno de Atenção Básica-Diabetes Mellitus. Brasília, DF: Ministério da Saúde; 2006.

5. Lyra R, Oliveira M, Lins D, Cavalcanti N. Prevenção do diabetes mellitus tipo 2. Arq Bras Endocrinol Metab 2006;50(2):239-49.

6. Asfaha S, Padwal R. Antihypertensive drugs and incidence of type 2 diabetes: evidence and implications for clinical practice. Curr Hypertens Rep 2005;7(5):314-22.

7. Mancia G, Grassi G, Zanchetti A. New-onset diabetes and antihypertensive drugs. J Hypertens 2006;24(1):3-10.

8. Barzilay JI, Davis BR, Cutler JA, Pressel SL, Whelton PK, Basile J, et al. Fasting glucose levels and incident diabetes mellitus in older nondiabetic adults randomized to receive 3 different classes of antihypertensive treatment: a report from the Antihypertensive and Lipid-Lowering Treatment to Prevent Heart Attack Trial (ALLHAT). Arch Intern Med 2006;166(20):2191-201.

9. Fráguas R, Soares SMSR, Bronstein MD. Depressão e diabetes mellitus. Rev Psiquiatr Clín 2009;36(3):93-9.

10. Rubin RR, Ma Y, Marrero DG, Peyrot M, Barrett-Connor EL, Kanh SE, et al. Elevated depression symptoms, antidepressant medicine use, and risk of developing diabetes during the diabetes prevention program. Diabetes Care 2008;31(3):420-6.
11. Araújo MFM, Gonçalves TC, Damasceno MMC, Caetano JA. Aderência de diabéticos ao tratamento medicamentoso com hipoglicemiantes orais. Esc Anna Nery Rev Enferm 2010;14(2):361-7.

12. Pascali PM. Monitorização da glicemia. In: Grossi, SAA, Pascali PM, organizadores. Cuidados de enfermagem em diabetes mellitus. São Paulo: Sociedade Brasileira de Diabetes; 2009. p.41-55.

13. Cervi A, Franceschini SCC, Priore SE. Análise crítica do uso do índice de massa corporal para idosos. Rev Nutr 2005;18(6):765-775.

14. Flores VB, Benvegnú LA. Perfil de utilização de medicamentos em idosos da zona urbana de Santa Rosa, Rio Grande do Sul, Brasil. Cad Saúde Pública 2008;24(6):1439-46.

15. Grillo MFF, Gorini MIPC. Caracterização de pessoas com diabetes mellitus tipo 2. Rev Bras Enferm 2007;60(1):49-54.

16. Moreno AH, Nogueira EP, Perez MPMS, Lima LRO. Atenção farmacêutica na prevenção de interações medicamentosas em hipertensos. Rev Inst Ciênc Saúde 2007;25(4):373-7

17. Abuissa H, Jones PG, Marso SP, $\mathrm{O}^{\prime}$ Keefe Junior JH. Angiotensin-converting enzyme inhibitors or angiotensin receptor blockers for prevention of type 2 diabetes: a metaanalysis of randomized clinical trials. J Am Coll Cardiol 2005;46(5):821-6.

18. Galletti F, Strazzullo P. Involvement of the renin-angiotensin system in obesity: older and newer pathways. Nutr Metab Cardiovasc Dis 2007;17(10):699-704.

19. Drouillet $\mathrm{P}$, Balkau B, Charles MA, Vol S, Bedouet $M$, Ducimetiére $\mathrm{P}$, et al. Calcium consumption and insulin resistance syndrome parameters. Data from the Epidemiological Study on the Insulin Resistance Syndrome (DESIR). Nutr Metab Cardiovasc Dis 2007;17(7):486-92.

20. Pittas AG, Harris SS, Stark PC, Dawson-Hughes B. The effects of calcium and vitamin $\mathrm{D}$ supplementation on blood glucose and markers of inflammation in nondiabetic adults. Diabetes care 2007;30(4):980-6. 